This is a preprint of an article in press in Proceedings IWOTA 2010 as a special volume of Operator Theory: Advances and Applications (OT) series Birkhauser Verlag.

\title{
Canonical eigenvalue distribution of multilevel block Toeplitz sequences with non-Hermitian symbols
}

\author{
Marco Donatelli, Maya Neytcheva and Stefano Serra-Capizzano
}

\begin{abstract}
Let $f: I_{k} \rightarrow \mathcal{M}_{s}$ be a bounded symbol with $I_{k}=(-\pi, \pi)^{k}$ and $\mathcal{M}_{s}$ be the linear space of the complex $s \times s$ matrices, $k, s \geq 1$. We consider the sequence of matrices $\left\{T_{n}(f)\right\}$, where $n=\left(n_{1}, \ldots, n_{k}\right), n_{j}$ positive integers, $j=1 \ldots, k$. Let $T_{n}(f)$ denote the multilevel block Toeplitz matrix of size $\widehat{n} s$, $\widehat{n}=\prod_{j=1}^{k} n_{j}$, constructed in the standard way by using the Fourier coefficients of the symbol $f$. If $f$ is Hermitian almost everywhere, then it is well known that $\left\{T_{n}(f)\right\}$ admits the canonical eigenvalue distribution with the eigenvalue symbol given exactly by $f$ that is $\left\{T_{n}(f)\right\} \sim_{\lambda}\left(f, I_{k}\right)$. When $s=1$, thanks to the work of Tilli, more about the spectrum is known, independently of the regularity of $f$ and relying only on the topological features of $R(f), R(f)$ being the essential range of $f$. More precisely, if $R(f)$ has empty interior and does not disconnect the complex plane, then $\left\{T_{n}(f)\right\} \sim_{\lambda}\left(f, I_{k}\right)$. Here we generalize the latter result for the case where the role of $R(f)$ is played by $\bigcup_{j=1}^{s} R\left(\lambda_{j}(f)\right), \lambda_{j}(f), j=1, \ldots, s$, being the eigenvalues of the matrixvalued symbol $f$. The result is extended to the algebra generated by Toeplitz sequences with bounded symbols. The theoretical findings are confirmed by numerical experiments, which illustrate their practical usefulness.
\end{abstract}

Mathematics Subject Classification (2000). Primary 47B35, Secondary 15A18. Keywords. matrix sequence, joint eigenvalue distribution, Toeplitz matrix.

\section{Introduction and basic notations}

Let $\mathcal{M}_{s}$ be the linear space of the complex $s \times s$ matrices and let $f$ be a $\mathcal{M}_{s^{-}}$ valued function of $k$ variables, integrable on the $k$-dimensional cube $I_{k}:=(-\pi, \pi)^{k}$. Throughout, the symbol $f_{I_{k}}$ stands for $(2 \pi)^{-k} \int_{I_{k}}$ and the symbol $L^{p}(s)$ stands for $L^{p}\left(I^{k},(2 \pi)^{-k} d x, \mathcal{M}_{s}\right), p \in[1, \infty]$, that is $f \in L^{p}(s)$ if and only if every scalar

The work of the first and the third authors is partly supported by MIUR grant no. 20083KLJEZ; the work of the second and the third authors is partly supported by a grant for visiting researchers of the Mathematics and Computer Science section of the Faculty of Science and Technology, Uppsala University, Sweden, 2008-2010. 
function $f_{i, j} \in L^{p}\left(I^{k},(2 \pi)^{-k} d x, \mathbb{C}\right)$. The Fourier coefficients of $f$, given by $\widehat{f}_{j}:=$ $f_{I_{k}} f(t) e^{-\mathbf{i}\langle j, t\rangle} d t \in \mathcal{M}_{s}, \mathbf{i}^{2}=-1, j \in \mathbb{Z}^{k},\langle j, t\rangle=\sum_{l=1}^{k} j_{l} t_{l}$, are the entries of the $k$-level Toeplitz matrices generated by $f$. More precisely, if $n=\left(n_{1}, \ldots, n_{k}\right)$ is a $k$-index with positive entries, then $T_{n}(f)$ denotes the matrix of order $\widehat{n} s$ given by $T_{n}(f)=\sum_{\left|j_{1}\right|<n_{1}} \cdots \sum_{\left|j_{k}\right|<n_{k}}\left[J_{n_{1}}^{\left(j_{1}\right)} \otimes \cdots \otimes J_{n_{k}}^{\left(j_{k}\right)}\right] \otimes \widehat{f}_{\left(j_{1}, \ldots, j_{k}\right)}$ (throughout, we let $\left.\widehat{n}:=\prod_{i=1}^{k} n_{i}\right)$. In this case, we say that the sequence $\left\{T_{n}(f)\right\}$ is generated by $f$. In the above equation, $\otimes$ denotes the tensor product, while $J_{t}^{(l)}$ denotes the matrix of order $t$ whose $(i, j)$ entry equals 1 if $j-i=l$ and equals zero otherwise. In the following, the notation $n \rightarrow \infty$ indicates that $\min _{1 \leq r \leq k} n_{r} \rightarrow \infty$ and $\operatorname{tr}(A)$ denotes the trace of a square matrix $A$, that is the sum of its eigenvalues.

In this paper we consider the global behavior of the spectrum of the sequence $\left\{T_{n}(f)\right\}$ and more precisely, its canonical distribution in the sense of Szegö (cf. [7], see also the rich book [3] and references therein for the standard scalar-valued case where $s=1$ ). For the general case when $s$ is any positive integer, an earlier result is proved by Tilli [21], but imposing the strong restriction on the symbol $f$ to be Hermitian-valued almost everywhere (a.e.). For completeness we include the result.

Theorem 1.1 ([21]). Let $f \in L^{1}(s)$ be Hermitian-valued a.e. and let $\Lambda_{n}$ be the spectrum of $T_{n}(f)$, the set of all $\widehat{n} s$ eigenvalues of $T_{n}(f)$. (Note that these are real since $f$ is Hermitian-valued and the matrix $T_{n}(f)$ is Hermitian). Then, for every function $F \in \mathcal{C}_{0}(\mathbb{R})$ continuous with bounded support, the following asymptotic formula holds,

$$
\lim _{n \rightarrow \infty} \frac{1}{\widehat{n} s} \sum_{\lambda \in \Lambda_{n}} F(\lambda)=f_{I_{k}} \frac{1}{s} \operatorname{tr}(F(f(t))) d t
$$

that is, $\left\{T_{n}(f)\right\} \sim_{\lambda}\left(f, I_{k}\right)$.

In this paper we extend Theorem 1.1 to the case where $f$ is not necessarily Hermitian-valued and prove the following result.

Theorem 1.2. Let $f \in L^{\infty}(s)$ with eigenvalues $\lambda_{j}(f), j=1, \ldots, s$, and let $\Lambda_{n}$ be the spectrum of $T_{n}(f)$. Define the union of the essential ranges of the eigenvalues of $f$ as $R(f)=\bigcup_{j=1}^{s} R\left(\lambda_{j}(f)\right)$. If $R(f)$ has empty interior and does not disconnect the complex plane, then for every function $F \in \mathcal{C}_{0}(\mathbb{C})$, which is continuous and with bounded support, the following asymptotic formula holds,

$$
\lim _{n \rightarrow \infty} \frac{1}{\widehat{n} s} \sum_{\lambda \in \Lambda_{n}} F(\lambda)=\int_{I_{k}} \frac{1}{s} \operatorname{tr}(F(f(t))) d t
$$

that is, $\left\{T_{n}(f)\right\} \sim_{\lambda}\left(f, I_{k}\right)$.

Theorem 1.2 generalizes a result by Tilli [22] to the matrix-valued case including the scalar-valued case $(s=1)$. We note that the same proof with obvious variations can be used for matrix sequences belonging to the algebra, generated by all Toeplitz sequences with bounded symbols (see also $[8,17]$ ). 
The paper is organized as follows. In Section 2 we introduce preliminary definitions and tools. In Section 3 we extend to the block case some technical instruments, based on the Mergelyan theorem (refer to [10]), that we employ in Section 4 in order to prove Theorem 1.2 and its generalization to the case of the block Toeplitz algebra. In Section 5 we include numerical illustrations and indicate the practical usefulness of our theoretical findings. The conclusions in Section 6, including a few open questions, finalize the paper.

\section{Preliminary definitions and tools}

We start with a general definition of an eigenvalue distribution and of weak/strong clustering for a matrix sequence, and recall the notion of essential range, which plays an important role in the study of the asymptotic properties of the spectrum.

For a matrix $A \in \mathbb{C}^{n \times n}$ with singular values $\sigma_{1}(A), \ldots, \sigma_{n}(A)$, and $p \in[1, \infty]$ we define $\|A\|_{p}$, the Schatten $p$-norm of $A$, to be the $\ell^{p}$ norm of the vector of the singular values $\|A\|_{p}=\left[\sum_{k=1}^{n}\left(\sigma_{k}(A)\right)^{p}\right]^{\frac{1}{p}}$. We also consider the norm $\|\cdot\|_{1}$ which is also known as the trace norm, the norm $\|\cdot\|_{2}$ also known in the numerical analysis community as the Frobenius norm, and the spectral norm $\|\cdot\|_{\infty}$, which is equal to the operator norm. Now, if $\lambda_{j}(A), j=1, \ldots, n$ are the eigenvalues of $A$, and $F$ is a function defined on $\mathbb{C}$, we define the mean

$$
\Sigma_{\lambda}(F, A):=\frac{1}{n} \sum_{j=1}^{n} F\left(\lambda_{j}(A)\right)=\frac{1}{n} \sum_{\lambda \in \Lambda_{n}} F(\lambda) .
$$

Analogously, we define $\Sigma_{\sigma}(F, A)$ with the singular values replacing the eigenvalues.

Definition 2.1. Let $\mathcal{C}_{0}(\mathbb{C})$ be the set of continuous functions with bounded support defined over the complex field, $k$ be a positive integer, and $\theta$ be a $s \times s$ matrixvalued measurable function defined on a set $G \subset \mathbb{R}^{k}$ of finite and positive Lebesgue measure $m(G)$. Here $G$ is equal to $I_{k}$ and a function is considered to be measurable if and only if the component functions are.

(i) A matrix sequence $\left\{A_{n}\right\}$ is said to be distributed (in the sense of the eigenvalues) as the pair $(\theta, G)$, that is $\left\{A_{n}\right\} \sim_{\lambda}(\theta, G)$, or to have the distribution function $\theta$, if for any $F \in \mathcal{C}_{0}(\mathbb{C})$, the following limit relation holds

$$
\lim _{n \rightarrow \infty} \Sigma_{\lambda}\left(F, A_{n}\right)=\int_{G} \frac{\sum_{j=1}^{s} F\left(\lambda_{j}(\theta(t))\right)}{s} d t,=\int_{G} \frac{\operatorname{tr}(F(\theta(t)))}{s} d t,
$$

where $\lambda_{i}(\theta(t))$ are the eigenvalues of the matrix $\theta(t)$ and $\underset{G}{f}=\frac{1}{m(G)} \int_{G}$.

(ii) If (3) holds for every $F \in \mathcal{C}_{0}\left(\mathbb{R}_{0}^{+}\right)$in place of $F \in \mathcal{C}_{0}(\mathbb{C})$, with the singular values $\sigma_{j}\left(A_{n}\right), j=1, \ldots, n$, in place of the eigenvalues, and with $|\theta(t)|=$ $\left(\theta(t)^{*} \theta(t)\right)^{1 / 2}$ in place of $\theta(t)$, we say that $\left\{A_{n}\right\} \sim_{\sigma}(\theta, G)$ or that the matrix 
sequence $\left\{A_{n}\right\}$ is distributed in the sense of the singular values as the pair $(\theta, G):$ more specifically, for every $F \in \mathcal{C}_{0}\left(\mathbb{R}_{0}^{+}\right)$we have

$$
\lim _{n \rightarrow \infty} \Sigma_{\sigma}\left(F, A_{n}\right)=\int_{G} \frac{\sum_{j=1}^{s} F\left(\sigma_{j}(\theta(t))\right)}{s} d t=\int_{G} \frac{\operatorname{tr}(F(|\theta(t)|))}{s} d t .
$$

Definition 2.2. A matrix sequence $\left\{A_{n}\right\}$ is said to be strongly clustered at $r \in \mathbb{C}$ (in the eigenvalue sense), if for any $\epsilon>0$ the number of the eigenvalues of $A_{n}$ off the disc

$$
D(r, \epsilon):=\{z:|z-r|<\epsilon\},
$$

can be bounded by a constant $q_{\epsilon}$ possibly depending on $\epsilon$, but not on $n$. In other words

$$
q_{\epsilon}(n, r):=\#\left\{j: \lambda_{j}\left(A_{n}\right) \notin D(r, \epsilon)\right\}=O(1), \quad n \rightarrow \infty .
$$

If each $A_{n}$ has only real eigenvalues (at least for large $n$ ) then we may assume that $r$ is real and that the disc $D(r, \epsilon)$ is the interval $(r-\epsilon, r+\epsilon)$. A matrix sequence $\left\{A_{n}\right\}$ is said to be strongly clustered at a nonempty closed set $S \subset \mathbb{C}$ (in the eigenvalue sense) if for any $\epsilon>0$

$$
q_{\epsilon}(n, S):=\#\left\{j: \lambda_{j}\left(A_{n}\right) \notin D(S, \epsilon)\right\}=O(1), \quad n \rightarrow \infty,
$$

where $D(S, \epsilon):=\cup_{r \in S} D(r, \epsilon)$ is the $\epsilon$-neighborhood of $S$. If each $A_{n}$ has only real eigenvalues, then $S$ is a nonempty closed subset of $\mathbb{R}$. We replace the term "strongly" by "weakly", if

$$
q_{\epsilon}(n, r)=o(n), \quad\left(q_{\epsilon}(n, S)=o(n)\right), \quad n \rightarrow \infty,
$$

in the case of a point $r$ or a closed set $S$. If $S$ is not connected, then its disjoint parts are called sub-clusters. By replacing 'eigenvalues' with 'singular values' we obtain all the corresponding definitions for singular values.

It is clear that $\left\{A_{n}\right\} \sim_{\lambda}(\theta, G)$, with $\theta \equiv r$ equal to a constant function if and only if $\left\{A_{n}\right\}$ is weakly clustered at $r \in \mathbb{C}$. For more results and relations between the notions of equal distribution, equal localization, spectral distribution, spectral clustering etc., see $[14$, Section 4$]$.

Definition 2.3. Given a measurable complex-valued function $\theta$ defined on a Lebesgue measurable set $G$, the essential range of $\theta$ is the set $R(\theta)$ of points $r \in \mathbb{C}$ such that, for every $\epsilon>0$, the Lebesgue measure of the set $\theta^{(-1)}(D(r, \epsilon)):=\{t \in G: \theta(t) \epsilon$ $D(r, \epsilon)\}$ is positive, with $D(r, \epsilon)$ as in (5). The function $\theta$ is essentially bounded if its essential range is bounded. Furthermore, if $\theta$ is real-valued, then the essential supremum (infimum) is defined as the supremum (infimum) of its essential range. Finally, if the function $\theta$ is $s \times s$ matrix-valued and measurable, then the essential range of $\theta$, denoted again by $R(\theta)$, is the union of the essential ranges of the complex-valued eigenvalues $\lambda_{j}(\theta), j=1, \ldots, s$, that is $R(\theta)=\bigcup_{j=1}^{s} R\left(\lambda_{j}(\theta)\right)$.

We note that $R(\theta)$ is clearly a closed set and, thus, its complement is open. Moreover, if $\left\{A_{n}\right\}$ is a matrix sequence distributed as $\theta$ in the sense of eigenvalues, then $R(\theta)$ is a weak cluster for $\left\{A_{n}\right\}$. 
Eigenvalue distribution of non-Hermitian multilevel block Toeplitz matrices 5

\section{Further tools for general matrix sequences and main results}

The basic ideas used in this section originate in [22] and [6], where the same questions are considered in a scalar Toeplitz context and in a scalar Jacobi context, respectively. We consider now how to extend these results to the case, where the symbol is matrix-valued, that is when $s>1$.

Theorem 3.1. Let $\left\{A_{n}\right\}$ be a matrix sequence and $S$ be a subset of $\mathbb{C}$. Assume that the following assumptions hold:

(a1) $S$ is a compact set and $\mathbb{C} \backslash S$ is connected;

(a2) the matrix sequence $\left\{A_{n}\right\}$ is weakly clustered at $S$;

(a3) the spectra $\Lambda_{n}$ of $A_{n}$ are uniformly bounded, i.e., $\exists C \in \mathbb{R}^{+}$such that $|\lambda|<C$, $\lambda \in \Lambda_{n}$, for all $n$;

(a4) there exists a $s \times s$ matrix-valued function $\theta$, which is measurable, bounded, and defined on a set $G$ of positive and finite Lebesgue measure, such that, for every positive integer $L$, we have $\lim _{n \rightarrow \infty} \frac{\operatorname{tr}\left(A_{n}^{L}\right)}{n}=\int_{G} \frac{\operatorname{tr}\left(\theta^{L}(t)\right)}{s} d t$, i.e., relation

(3) holds with $F$ being any polynomial of an arbitrary fixed degree;

(a5) the essential range of $\theta$ is contained in $S$.

Then relation (3) is true for every continuous function $F$ with bounded support, which is holomorphic in the interior of $S$. If also the interior of $S$ is empty, then the sequence $\left\{A_{n}\right\}$ is distributed as $(\theta, G)$, in the sense of the eigenvalues.

Proof. The proof follows that of Theorem 2.2 in [6]. Let $F$ be continuous over $S$ and holomorphic in its interior. By Mergelyan's Theorem [10], for every $\epsilon>0$, we can find a polynomial $P$ such that for every $z \in S,|P(z)-F(z)| \leq \epsilon$. Since $R(\theta)$ is contained in $S$, it is clear that $\left|P\left(\lambda_{j}(\theta(t))\right)-F\left(\lambda_{j}(\theta(t))\right)\right| \leq \epsilon, j=1, \ldots, s$ a.e. in its domain $G$ so that $|\operatorname{tr}(F(\theta(t)))-\operatorname{tr}(P(\theta(t)))| \leq s \epsilon$. Therefore,

$$
\left|f_{G} \frac{\operatorname{tr}(F(\theta(t)))}{s} d t-\int_{G} \frac{\operatorname{tr}(P(\theta(t)))}{s} d t\right| \leq f_{G} \epsilon d t=\epsilon .
$$

Next, we consider the left-hand side of (3). By the definition of clustering, for any fixed $\epsilon^{\prime}>0$, we have

$$
\#\left\{\lambda \in \Sigma_{n},|\lambda-z| \geq \epsilon^{\prime}, \forall z \in S\right\}=\#\left\{\lambda \in \Sigma_{n}, \lambda \notin D\left(S, \epsilon^{\prime}\right)\right\}=o(n) .
$$

Moreover, by the assumption of the uniform boundedness of $\Sigma_{n}$, the bound $|\lambda|<$ $C$ holds for every $\lambda \in \Sigma_{n}$ with a constant $C$, independent of $n$. Therefore, by extending $F$ outside $S$ in such a way that it is continuous with a bounded support, we infer

$$
\left|\frac{1}{n} \sum_{\lambda \in \Sigma_{n}, \lambda \notin D\left(S, \epsilon^{\prime}\right)} F(\lambda)\right| \leq \frac{M}{n} \#\left\{\lambda \in \Sigma_{n}, \lambda \notin D\left(S, \epsilon^{\prime}\right)\right\}=o(1),
$$




$$
\left|\frac{1}{n} \sum_{\lambda \in \Sigma_{n}, \lambda \notin D\left(S, \epsilon^{\prime}\right)} P(\lambda)\right| \leq \frac{M}{n} \#\left\{\lambda \in \Sigma_{n}, \lambda \notin D\left(S, \epsilon^{\prime}\right)\right\}=o(1),
$$

with $M=\max \left(\|F\|_{\infty},\|P\|_{\infty}\right)$, and infinity norms taken over $\{z \in \mathbb{C},|z| \leq C\}$. Consequently, by setting $\Delta=\left|\Sigma_{\lambda}\left(F-P, A_{n}\right)\right|$, we deduce that

$$
\begin{aligned}
\Delta & =\left|\frac{1}{n} \sum_{\lambda \in \Sigma_{n}}(F(\lambda)-P(\lambda))\right| \leq \frac{1}{n} \sum_{\lambda \in \Sigma_{n}}|F(\lambda)-P(\lambda)| \\
& =\frac{1}{n} \sum_{\lambda \in \Sigma_{n}, \lambda \in D\left(S, \epsilon^{\prime}\right)}|F(\lambda)-P(\lambda)|+\frac{1}{n} \sum_{\lambda \in \Sigma_{n}, \lambda \notin D\left(S, \epsilon^{\prime}\right)}|F(\lambda)-P(\lambda)| \\
& \leq \frac{1}{n} \sum_{\lambda \in \Sigma_{n}, \lambda \in D\left(S, \epsilon^{\prime}\right)}|F(\lambda)-P(\lambda)|+o(1) \\
& =\frac{1}{n} \sum_{\lambda \in \Sigma_{n}, \lambda \in S}|F(\lambda)-P(\lambda)|+\frac{1}{n} \sum_{\lambda \in \Sigma_{n}, \lambda \in D\left(S, \epsilon^{\prime}\right) \backslash S}|F(\lambda)-P(\lambda)|+o(1) .
\end{aligned}
$$

For $\lambda \in S$ we use the relation $|F(\lambda)-P(\lambda)| \leq \epsilon$. For $\lambda \in D\left(S, \epsilon^{\prime}\right) \backslash S$, we see that $|F(\lambda)-P(\lambda)| \leq\left|F(\lambda)-F\left(\lambda^{\prime}\right)\right|+\left|F\left(\lambda^{\prime}\right)-P\left(\lambda^{\prime}\right)\right|+\left|P\left(\lambda^{\prime}\right)-P(\lambda)\right|, \quad\left|\lambda-\lambda^{\prime}\right|<\epsilon^{\prime}, \quad \lambda^{\prime} \in S$, thus, $|F(\lambda)-P(\lambda)| \leq c_{1}\left(\epsilon^{\prime}\right)+\epsilon+c_{2}\left(\epsilon, \epsilon^{\prime}\right) \equiv \theta\left(\epsilon, \epsilon^{\prime}\right)$ with

$$
\lim _{\epsilon \rightarrow 0} \lim _{\epsilon^{\prime} \rightarrow 0} \theta\left(\epsilon, \epsilon^{\prime}\right)=0 \text {. }
$$

Hence

$$
\Delta \leq \epsilon+\theta\left(\epsilon, \epsilon^{\prime}\right)+o(1) .
$$

Furthermore, from the hypothesis of the theorem, there holds

$$
\lim _{n \rightarrow \infty} \Sigma_{\lambda}\left(P, A_{n}\right)=\int_{G} \frac{\operatorname{tr}(P(\theta(t)))}{s} d t .
$$

Since $\epsilon$ and $\epsilon^{\prime}$ are arbitrary, it is clear that relations (7)-(10) imply that (3) holds for $F$ as well. Finally, when $S$ has an empty interior, we have no restriction on $F$ except for being continuous with a bounded support, and therefore what we have proved is equivalent to $\left\{A_{n}\right\} \sim_{\lambda}(\theta, G)$.

Next, we show that the hypotheses (a3) and (a4) (or a slightly stronger form of it) imply (a1), (a2), and (a3) for the set $S$ defined by "filling in" the essential range of the function $\theta$ from (a4) (or its strengthened version). This shows that, when the set $R(\theta)$ has an empty interior, the matrix sequence has the desired distribution.

Here, "filling in" means taking the "Area" in the following sense:

Definition 3.2. Let $K$ be a compact subset of $\mathbb{C}$. We define Area $(K)$ as

$$
\operatorname{Area}(K)=\mathbb{C} \backslash U,
$$

where $U$ is the (unique) unbounded connected component of $\mathbb{C} \backslash K$. 
Theorem 3.3. Let $\left\{A_{n}\right\}$ be a matrix sequence. If

(b1) the spectra $\Lambda_{n}$ of $A_{n}$ are uniformly bounded, i.e., $\exists C \in \mathbb{R}^{+}$such that $|\lambda|<C$, $\lambda \in \Lambda_{n}$, for all $n$;

(b2) there exists a $s \times s$ matrix-valued function $\theta$ - measurable, bounded, and defined on a set $G$ of positive and finite Lebesgue measure, such that, for all positive integers $L$ and $l$, we have $\lim _{n \rightarrow \infty} \frac{\operatorname{tr}\left(\left(A_{n}^{*}\right)^{l} A_{n}^{L}\right)}{n}=f_{G} \frac{\operatorname{tr}\left(\overline{\theta^{l}(t)} \theta^{L}(t)\right)}{s} d t$;

then $R(\theta)$ is compact, the matrix sequence $\left\{A_{n}\right\}$ is weakly clustered at Area $(R(\theta))$, and relation (3) is true for every continuous function $F$ with bounded support, which is holomorphic in the interior of $S=\operatorname{Area}(R(\theta))$.

If it is also true that $\mathbb{C} \backslash R(\theta)$ is connected and the interior of $R(\theta)$ is empty then the sequence $\left\{A_{n}\right\}$ is distributed as $(\theta, G)$, in the sense of the eigenvalues.

Proof. Since $\theta$ is bounded, any of its eigenvalues $\lambda_{j}(\theta)$ is bounded, $j=1, \ldots, s$ so that $R(\theta)=\bigcup_{j=1}^{s} R\left(\lambda_{j}(\theta)\right)$ is bounded. Consequently, the set $R(\theta)$ is compact, since the essential range is always closed. Hence we can define $S=\operatorname{Area}(R(\theta))$ according to Definition 3.2.

We prove that $S$ is a weak cluster for the spectra of $\left\{A_{n}\right\}$. First, we notice that the compact set $S_{C}=\{z \in \mathbb{C}:|z| \leq C\}$ is a strong cluster for the spectra of $\left\{A_{n}\right\}$ since by (b1) it contains all the eigenvalues. Moreover $C$ can be chosen such that $S_{C}$ contains $S$. Therefore, to prove that $S$ is a weak cluster for $\left\{A_{n}\right\}$ it suffices to prove that, for every $\epsilon>0$ the compact set $S_{C} \backslash D(S, \epsilon)$ contains at most only $o(n)$ eigenvalues, with $D(S, \epsilon)$ as in Definition 2.2. By compactness, for any $\delta>0$, there exists a finite covering of $S_{C} \backslash D(S, \epsilon)$ made of balls $D(z, \delta)$, $z \in S_{C} \backslash S$ with $D(z, \delta) \cap S=\emptyset$, and so, it suffices to show that, for a particular $\delta$, at most $o(n)$ eigenvalues lie in $D(z, \delta)$. Let $F(t)$ be the characteristic function of the compact set $\overline{D(z, \delta)}$. Then restricting our attention to the compact set $\overline{D(z, \delta)} \cup S$, Mergelyan's theorem implies that for each $\epsilon>0$ there exists a polynomial $P$ such that $|F(t)-P(t)|$ is bounded by $\epsilon$ on $\overline{D(z, \delta)} \cup S$.

Therefore $\left|P\left(\lambda_{j}(\theta(t))\right)\right| \leq \epsilon$ a.e. in its domain for every $j=1, \ldots, s$. However the latter does not guarantee

$$
\|P(\theta(t))\|_{2}<l(\epsilon)
$$

a.e. for some $l(\epsilon)$ converging to zero as $\epsilon$ converges to zero (it would be trivially true if $\theta(t)$ is normal a.e. that is $\theta^{*}(t) \theta(t)=\theta(t) \theta^{*}(t)$ a.e., since, in that case $P(\theta(t))$ is also normal a.e. and $\|P(\theta(t))\|_{2}=\left(\sum_{j=1}^{s}\left|\lambda_{j}(P(\theta(t)))\right|^{2}\right)^{1 / 2}=$ $\left(\sum_{j=1}^{s}\left|P\left(\lambda_{j}(\theta(t))\right)\right|^{2}\right)^{1 / 2}<\sqrt{s} \epsilon$ a.e. $)$. The condition (11) on the Frobenius norm is essential in the proof as we will see later. Thus, in order to fulfill it, starting from the Schur normal form of $\theta(t)$, we define a new polynomial $P_{k}$ satisfying the claim and at the same time approximating $F(t)$ over $\overline{D(z, \delta)} \cup S$. We write $\theta(t)=U(t) T(t) U^{*}(t)$ with $U(t)$ unitary a.e. and $T(t)=\Lambda(t)+R(t)$ upper triangular, where $\Lambda(t)=\operatorname{diag}_{j=1, \ldots, s} \lambda_{j}(\theta(t))$ and $R(t)$ strictly upper triangular and 
bounded. Clearly, $P(\theta(t))=U(t)(P(\Lambda(t))+\tilde{R}(t)) U^{*}(t)$ with $\tilde{R}(t)$ still strictly upper triangular and bounded. Let $k$ be any integer larger than $s-1$. Then $\tilde{R}^{k}(t)=0$ since $\tilde{R}(t)$ is of order $s$ and strictly upper triangular (so, nilpotent). Consequently, by defining $P_{k}(y)=p^{k}(y)$, we have

$$
P_{k}(\theta(t))=U(t)(P(\Lambda(t))+\tilde{R}(t))^{k} U^{*}(t)
$$

so that

$$
(P(\Lambda(t))+\tilde{R}(t))^{k}=\sum_{j=k-s+1}^{k}\left(\begin{array}{c}
k \\
j
\end{array}\right) p^{j}(\Lambda(t)) \tilde{R}^{k-j}(t) .
$$

Of course $\left\|P_{k}(\theta(t))\right\|_{2}=\left\|(P(\Lambda(t))+\tilde{R}(t))^{k}\right\|_{2}$ and thanks to (12) and to the boundedness of the symbol $\theta$, we can choose $k$ independent of $\epsilon$ and $t$ so that

$$
\left\|P_{k}(\theta(t))\right\|_{2}^{2}<s \epsilon
$$

a.e., which is the desired relation (11) with $l(\epsilon)=s \epsilon$.

$$
\begin{aligned}
(1-\epsilon)^{k} \gamma_{n}(z, \delta) & \leq \sum_{i=1}^{n} F\left(\lambda_{i}\right)\left|P_{k}\left(\lambda_{i}\right)\right| \\
& \leq\left(\sum_{i=1}^{n} F^{2}\left(\lambda_{i}\right)\right)^{1 / 2}\left(\sum_{i=1}^{n}\left|P_{k}\left(\lambda_{i}\right)\right|^{2}\right)^{1 / 2} \\
& =\left(\sum_{i=1}^{n} F\left(\lambda_{i}\right)\right)^{1 / 2}\left(\sum_{i=1}^{n}\left|P_{k}\left(\lambda_{i}\right)\right|^{2}\right)^{1 / 2} \\
& =\left(\gamma_{n}(z, \delta)\right)^{1 / 2}\left(\sum_{i=1}^{n}\left|P_{k}\left(\lambda_{i}\right)\right|^{2}\right)^{1 / 2} \\
& \leq\left(\gamma_{n}(z, \delta)\right)^{1 / 2}\left\|P_{k}\left(A_{n}\right)\right\|_{2} \\
& =\left(\gamma_{n}(z, \delta)\right)^{1 / 2}\left(\operatorname{tr}\left(P_{k}^{*}\left(A_{n}\right) P_{k}\left(A_{n}\right)\right)\right)^{1 / 2} \\
& =\left(\gamma_{n}(z, \delta)\right)^{1 / 2}\left(\operatorname{tr}\left(\sum_{l, L=0}^{M} \overline{c_{l}} c_{L}\left(A_{n}^{*}\right)^{l} A_{n}^{L}\right)\right)^{1 / 2} \\
& =\left(\gamma_{n}(z, \delta)\right)^{1 / 2}\left(\sum_{l, L=0}^{M} \overline{c_{l}} c_{L} \operatorname{tr}\left(\left(A_{n}^{*}\right)^{l} A_{n}^{L}\right)\right)^{1 / 2}
\end{aligned}
$$

where inequality (14) follows from the definition of $F$ and from the approximation properties of $P$, relation (15) is the Cauchy-Schwartz inequality, relations (16)(17) follow from the definitions of $F$ and $\gamma_{n}(z, \delta)$, (18) is a consequence of the Schur decomposition of $\theta(t)$ and of the unitary invariance of the Schatten norms, identities (19)-(21) follow from the entry-wise definition of the Schatten 2 norm 
(the Frobenius norm), from the monomial expansion of the polynomial $P$, and from the linearity of the trace.

Given $\epsilon_{2}>0$, we choose $\epsilon_{1}>0$ so that the inequality

$$
\epsilon_{1} \sum_{l, L=0}^{M}\left|c_{l}\right|\left|c_{L}\right| \leq \epsilon_{2}
$$

holds true and then we choose $N$ so that for $n>N$, inequality

$$
\left|\frac{\operatorname{tr}\left(\left(A_{n}^{*}\right)^{l} A_{n}^{L}\right)}{n}-f_{G} \frac{\operatorname{tr}\left(\overline{\theta^{l}(t)} \theta^{L}(t)\right)}{s} d t\right|<\epsilon_{1},
$$

is true. Hence, from (21) we obtain

$$
\begin{aligned}
(1-\epsilon)^{k} \gamma_{n}(z, \delta) & \leq\left(\gamma_{n}(z, \delta)\right)^{1 / 2}\left(n\left(\epsilon_{2}+\int_{G} \sum_{l, L=0}^{M} \overline{c_{l}} c_{L}\left(\frac{\operatorname{tr}\left(\overline{\theta^{l}(t)} \theta^{L}(t)\right)}{s}\right) d t\right)\right)^{1 / 2}(22) \\
& =\left(\gamma_{n}(z, \delta)\right)^{1 / 2}\left(n\left(\epsilon_{2}+\int_{G} \frac{\left\|P_{k}(\theta(t))\right\|^{2}}{s} d t\right)\right)^{1 / 2} \\
& \leq\left(\gamma_{n}(z, \delta)\right)^{1 / 2} n^{1 / 2}\left(\epsilon^{2}+\epsilon_{2}\right)^{1 / 2}
\end{aligned}
$$

where inequality (22) is assumption (b2). The latter two relations are again consequences of the monomial expansion of $P$ and of the crucial inequality (13), where $\epsilon_{2}$ is arbitrarily small. Therefore, by choosing $\epsilon_{2}=\epsilon^{2},(14)-(24)$ imply that, for $n$ sufficiently large,

$$
\gamma_{n}(z, \delta) \leq 2 n \epsilon^{2}(1-\epsilon)^{-2 k}
$$

which means that, since $k$ is chosen independent of $\epsilon, \gamma_{n}(z, \delta)=o(n)$.

Thus, hypotheses (a1)-(a5) of Theorem 3.1 hold with $S=\operatorname{Area}(R(\theta))$, which is necessarily compact and with connected complement. Consequently, the first conclusion in Theorem 3.1 holds. Finally, if $\mathbb{C} \backslash R(\theta)$ is connected and the interior of $R(\theta)$ is empty, then $\operatorname{Area}(R(\theta))=R(\theta)$ and, thus, all the hypotheses of Theorem 3.1 are satisfied, we conclude that the sequence $\left\{A_{n}\right\}$ is distributed in the sense of the eigenvalues as $(\theta, G)$.

Next, we present a second version of Theorem 3.3, replacing hypotheses (a1)(a5) by only (a3), (a4), and a condition on the Schatten $p$ norm for a certain $p$.

Theorem 3.4. Let $\left\{A_{n}\right\}$ be a matrix sequence. Assume that

(c1) the spectra $\Lambda_{n}$ of $A_{n}$ are uniformly bounded, i.e., $|\lambda|<C, \lambda \in \Lambda_{n}$, for all $n$;

(c2) there exists a $s \times s$ matrix-valued function $\theta$, which is measurable, bounded and defined over $G$ having positive and finite Lebesgue measure, such that for every positive integer $L$ there holds $\lim _{n \rightarrow \infty} \frac{\operatorname{tr}\left(A_{n}^{L}\right)}{n}=f_{G} \frac{\operatorname{tr}\left(\theta^{L}(t)\right)}{s} d t$; 
(c3) for every $n$ large enough, there exist a constant $\widehat{C}$ and a positive real number $p \in[1, \infty)$, independent of $n$, such that $\left\|P\left(A_{n}\right)\right\|_{p}^{p} \leq \widehat{C} n_{G} f(\theta(t)) \|_{p}^{p} d t$ for every fixed polynomial $P$ independent of $n$.

Then the matrix sequence $\left\{A_{n}\right\}$ is weakly clustered at Area $(R(\theta)):=\mathbb{C} \backslash U$ (see Definition 3.2) and relation (3) is true for every continuous function $F$ with bounded support which is holomorphic in the interior of $S=\operatorname{Area}(R(\theta))$.

Moreover, if

(c4) $\mathbb{C} \backslash R(\theta)$ is connected and the interior of $R(\theta)$ is empty,

then the sequence $\left\{A_{n}\right\}$ is distributed as $(\theta, G)$, in the sense of the eigenvalues.

Proof. The proof follows that of Theorem 3.3 until relation (14). Then, with $q$ being the the conjugate of $p$, i.e., $1 / q+1 / p=1$, we have

$$
\begin{aligned}
(1-\epsilon)^{k} \gamma_{n}(z, \delta) & \leq\left(\sum_{i=1}^{n} F^{q}\left(\lambda_{i}\right)\right)^{1 / q}\left(\sum_{i=1}^{n}\left|P\left(\lambda_{i}\right)\right|^{p}\right)^{1 / p} \\
& =\left(\sum_{i=1}^{n} F\left(\lambda_{i}\right)\right)^{1 / q}\left(\sum_{i=1}^{n}\left|P\left(\lambda_{i}\right)\right|^{p}\right)^{1 / p} \\
& =\left(\gamma_{n}(z, \delta)\right)^{1 / q}\left(\sum_{i=1}^{n}\left|P\left(\lambda_{i}\right)\right|^{p}\right)^{1 / p} \\
& \leq\left(\gamma_{n}(z, \delta)\right)^{1 / q}\left\|P\left(A_{n}\right)\right\|_{p} \\
& \leq\left(\gamma_{n}(z, \delta)\right)^{1 / q}\left(\frac{\widehat{C} n}{m(G)} \int_{G}\|P(\theta(t))\|_{p}^{p} d t\right)^{1 / p} \\
& \leq\left(\gamma_{n}(z, \delta)\right)^{1 / q}(\widehat{C} n)^{1 / p} \epsilon,
\end{aligned}
$$

where relation (25) is the Hölder inequality, relations (26)-(27) follow from the definitions of $F$ and $\gamma_{n}(z, \delta),(28)$ holds due to the fact that, for any square matrix, the vector with the moduli of the eigenvalues is weakly-majorized by the vector of the singular values (see [1] for more details), inequality (29) is assumption (c3) (which holds for any polynomial of fixed degree), and finally inequality (30) follows from the approximation properties of $P$ over the area delimited by the range of $\theta$. Therefore,

$$
\gamma_{n}(z, \delta) \leq \widehat{C} n \epsilon^{p}(1-\epsilon)^{-k p},
$$

and since $\epsilon$ is arbitrary we have the desired result, namely, $\gamma_{n}(z, \delta)=o(n)$.

The rest of the proof is the same as in Theorem 3.3.

The next result shows that the key assumption (c3) follows from the distribution in the singular value sense of $\left\{P\left(A_{n}\right)\right\}$ and that the latter is equivalent to the very same limit relation with only polynomial test functions. We mention here, that distribution results in the singular value sense (e.g., $[21,23,20,15,16])$ 
are much easier to obtain and to prove due to the higher stability of the singular values under perturbations (cf. [24]).

Theorem 3.5. Using the notation of Section 2, if the sequence $\left\{A_{n}\right\}$ is uniformly bounded in spectral norm then, (1), $\left\{A_{n}\right\} \sim_{\sigma}(\theta, G)$ is true whenever condition (4) holds for all polynomial test functions. Moreover, (2), if $\left\{P\left(A_{n}\right)\right\} \sim_{\sigma}(P(\theta), G)$ for every polynomial $P$ then the claim (c3) is true for every value $p \in[1, \infty)$, for every $\epsilon>0$ where $\widehat{C}=1+\epsilon$ and for $n$ larger than some fixed value $\bar{n}_{\epsilon}$.

Proof. The first claim is proved by using the fact that one can approximate any continuous function, defined on a compact set contained in the (positive) real line, by polynomials. The second claim follows from taking the function $z^{p}$, with positive $p$, as a test function and exploiting the limit relation from the assumption $\left\{P\left(A_{n}\right)\right\} \sim_{\sigma}(P(\theta), G)$. Indeed, the sequence $\left\{P\left(A_{n}\right)\right\}$ is uniformly bounded since $\left\{A_{n}\right\}$ is, so we can use as test functions continuous functions with no restriction on the support. Therefore, by definition (see (4)), $\left\{P\left(A_{n}\right)\right\} \sim_{\sigma}(P(\theta), G)$ implies that

$$
\lim _{n \rightarrow \infty} \frac{1}{n} \sum_{j=1}^{n} \sigma_{j}^{p}\left(P\left(A_{n}\right)\right)=f_{G}\|P(\theta(t))\|_{p}^{p} d t .
$$

Hence, by observing that $\sum_{j=1}^{n} \sigma_{j}^{p}\left(P\left(A_{n}\right)\right)$ is by definition $\left\|P\left(A_{n}\right)\right\|_{p}^{p}$ and by taking the limit, we see that, for every $\epsilon>0$, there exists an integer $\bar{n}_{\epsilon}$ such that

$$
\left\|P\left(A_{n}\right)\right\|_{p}^{p} \leq n \frac{1+\epsilon}{m(G)} \int_{G}\|P(\theta(t))\|_{p}^{p} d t, \quad \forall n \geq \bar{n}_{\epsilon} .
$$

The latter inequality coincides with (c3) with $\widehat{C}=1+\epsilon$ and $p \in[1, \infty)$.

We conclude this part of general tools by stating a simple but useful approximation result.

Theorem 3.6. Assume that two sequences $\left\{A_{n}\right\}$ and $\left\{B_{n}\right\}$ are given, with $\left\{A_{n}\right\}$ satisfying conditions (b1), (b2) or conditions (c1), (c2), (c3). In addition, we assume that the sequence $\left\{B_{n}\right\}$

(d1) is uniformly bounded in spectral norm and

(d2) $\left\|B_{n}-A_{n}\right\|_{1}=o(n)$ (that is $\left\{B_{n}\right\}$ approximates $\left\{A_{n}\right\}$ in trace norm).

Then $\left\{B_{n}\right\}$ satisfies the same conditions as $\left\{A_{n}\right\}$, that is, (b1), (b2) or (c1), (c2), (c3).

Proof. The assumption (d1) directly implies the equivalence between (b1) and (c1). Moreover the trace norm condition implies that any of the requirements (b2), (c2), (c3) is satisfied with $\left\{A_{n}\right\}$ if and only if the same requirement is satisfied for $\left\{B_{n}\right\}$ (we omit the details). 


\section{Proof of the main result for the case of block Toeplitz sequences and their algebra}

In this section we consider the case of Toeplitz sequences and prove Theorem 1.2, by exploiting the general tools developed above.

Proof. [Proof of Theorem 1.2] We make use of Theorem 3.4. (An alternative proof, similar to the approach used by Tilli and based on Theorem 3.3 is also possible, but requires more effort.)

Recall, that in [16, Section 3.3.1] it is proved that the algebra, generated by block Toeplitz sequences, is a subalgebra of the (block) GLT class so that, in particular, for every $\mathcal{M}_{s}$-valued symbol $f$ and for every polynomial of a given degree $P$ we have

$$
\left\{P\left(T_{n}(f)\right)\right\} \sim_{\sigma}\left(P(f), I_{k}\right) .
$$

Therefore, by invoking Theorem 3.5, we infer that the assumption (c3) is fulfilled for every $p$, when ever $f \in L^{\infty}(s)$. Furthermore, if $f \in L^{\infty}(s)$ then

$$
\left\|T_{n}(f)\right\|_{\infty} \leq \operatorname{esssup}_{t \in I_{k}}\|f(t)\|_{\infty}
$$

and hence assumption (c1) is satisfied with any constant $C>\operatorname{esssup}_{t \in I_{k}}\|f(t)\|_{\infty}$. Finally, a simple check (see e.g. $[2,17]$ ) shows that also (c2) is fulfilled. Thus, Theorem 3.4 can be applied and from that it follows that the matrix sequence $\left\{T_{n}(f)\right\}$ is weakly clustered at $\operatorname{Area}(R(f))$ (see Definition 3.2) and relation (2) is true for every continuous function $F$ with bounded support, which is holomorphic in the interior of $S=\operatorname{Area}(R(f))$. Furthermore, if $\mathbb{C} \backslash R(f)$ is connected and the interior of $R(f)$ is empty, then $\left\{T_{n}(f)\right\} \sim_{\lambda}\left(f, I_{k}\right)$ which concludes the proof of Theorem 1.2.

Finally, Theorem 3.6 allows us to generalize Theorem 1.2 to the algebra generated by block Toeplitz sequences with bounded symbols.

Theorem 4.1. Let $f_{\alpha, \beta} \in L^{\infty}(s)$ with $\alpha=1, \ldots, \rho, \beta=1, \ldots, q_{\alpha}, \rho, q_{\alpha}<\infty$. Let

$$
h=\sum_{\alpha=1}^{\rho} \prod_{\beta=1}^{q_{\alpha}} f_{\alpha, \beta},
$$

and consider the sequence $\left\{B_{n}\right\}$ with $B_{n}=\sum_{\alpha=1}^{\rho} \prod_{\beta=1}^{q_{\alpha}} T_{n}\left(f_{\alpha, \beta}\right)$. Then $\| B_{n}-$ $T_{n}(h) \|_{1}=o(\widehat{n}),\left\{A_{n}\right\}$ is weakly clustered at Area $(R(h))$ and relation

$$
\lim _{n \rightarrow \infty} \frac{1}{\widehat{n} s} \sum_{\lambda \in \Lambda_{n}} F(\lambda)=\int_{I_{k}} \frac{1}{s} \operatorname{tr}(F(h(t))) d t
$$

is true for every continuous function $F$ with bounded support, which is holomorphic in the interior of $S=$ Area $(R(h))$. Furthermore, when $\mathbb{C} \backslash R(h)$ is connected and the interior of $R(h)$ is empty, then $\left\{B_{n}\right\} \sim_{\lambda}\left(h, I_{k}\right)$.

Proof. The first claim, namely, $\left\|B_{n}-T_{n}(h)\right\|_{1}=o(\widehat{n})$, can be shown by simple computation (see, for instance, $[2,17]$ ). Further, the sequence $\left\{B_{n}\right\}$ is uniformly bounded in spectral norm since it belongs to the algebra generated by block Toeplitz sequences with bounded symbols. Therefore, Theorem 3.6 with $A_{n}=T_{n}(f)$ implies that conditions (c1), (c2), (c3) are satisfied with $\left\{B_{n}\right\}$, since 
the same conditions are satisfied by the sequence $\left\{A_{n}\right\}$ (see the proof of Theorem 1.2). Hence, the use of Theorem 3.4 allows to conclude the proof.

Remark 4.2. Observe that the case when $f(t)$ is diagonalizable by a constant transformation independent of $t$, is special in the sense that the Szegö-type distribution result holds under the milder assumption that every eigenvalue of $f$ (now a scalar complex-valued function) shows a range with empty interior and which does not disconnect the complex plane. This leaves open the question whether this weaker requirement is sufficient in general.

Other problems remain open. For instance, it would be interesting to extend the results of this paper to the case where the involved symbols are not necessarily bounded, but only integrable. Such situations occur when constructing preconditioners for Krylov methods. As already stressed in [16], in that case, the matrix theory-based approach seems more convenient, since the corresponding Toeplitz operators are not well defined when the symbols are not bounded.

Finally, it should be observed that the conditions on the symbol reported in Theorem 1.2 for the existence of a canonical distribution corresponding to the symbol are sufficient, but not necessary. In fact, for $f(t)=e^{-\mathbf{i} t}$ the range of $f$ is the complex unit circle, disconnecting the complex plane, while the eigenvalues are all equal to zero. However, if one takes the symbol $f(t)$ in (3.24), p.80 in [3] $\left(f(t)=e^{2 \mathbf{i} t}, t \in[0, \pi), f(t)=e^{-2 \mathbf{i} t}, t \in[\pi, 2 \pi)\right)$, then the range of $f$ is again the complex unit circle, which disconnects the complex plane, however the eigenvalues indeed distribute as determined by the symbol, as discussed in Example 5.39, pp. 167-169 in [3]. It would be instructive to understand how to discriminate between these two types of generating functions.

In the next section we illustrate numerically some of these issues.

\section{Numerical experiments}

The numerical experiments are divided into two parts. In the first part (Section 5.1) we consider examples, covered by the theoretical results from the previous section. As expected, the numerical results confirm the theoretical findings with the clustering, which is often of strong type.

An example of an important application, where the related problem can be formulated and analyzed using the spectral analysis of a Toeplitz sequence with non-Hermitian bounded symbol, is a signal restoration problem, where some of the sampling data are not available $(\mathrm{cf} .[12,4])$. For that problem, the numerical tests in [4], are exactly driven by the theory developed in the present paper.

In the second group of tests (Section 5.2) we consider block Toeplitz sequences with unbounded symbols, for which the Mergelyan Theorem cannot be used and our tools do not apply. The numerical tests, however, indicate that there is room for improving the theory by allowing unbounded symbols. The latter can be foreseen, provided the weaker assumption in Theorem 1.1, when dealing with Hermitian structures. 

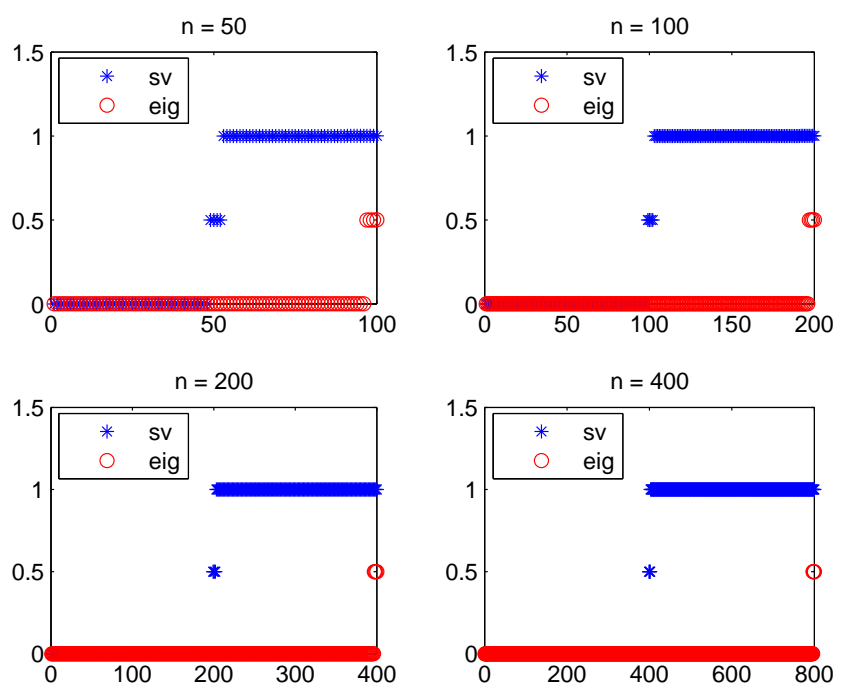

FiguRE 1. $T_{n}\left(f^{(1)}\right)$ : singular values and moduli of the eigenvalues in non decreasing order.

\subsection{Examples covered by the theory}

We considering first special classes of symbols $f$ where $s=2$ and

$$
\begin{gathered}
f(t)=Q(t) B(t) Q(t)^{T}, \quad t \in I_{1}, \\
Q(t)=\left(\begin{array}{cc}
\cos (t) & \sin (t) \\
-\sin (t) & \cos (t)
\end{array}\right)
\end{gathered}
$$

Below, $B(t)$ chosen in various ways, but having either constant eigenvalues, that is scalar functions independent of $t$, or eigenvalues with nicely behaved ranges. Thus, we expect a clustering of the eigenvalues of the corresponding block Toeplitz sequence, closely related to the shape of the spectra of the matrices.

Example 5.1 (Nonparametrized symbols). We choose

$$
B^{(1)}(t)=\left(\begin{array}{ll}
0 & 0 \\
1 & 0
\end{array}\right) \quad \text { and } \quad B^{(2)}(t)=\left(\begin{array}{cc}
0 & 0 \\
1 & 1
\end{array}\right)
$$

In that case we find that the singular values of the matrix $T_{n}\left(f^{(1)}\right)$ are divided into two sub-clusters of the same size (one at zero, the other at one), while its eigenvalues are clustered at zero (Figure 1). Interestingly enough, the eigenvalues clustered at zero are real, while the outliers are just four, independently of the size $n$, and lie on the imaginary axis (Figure $2(\mathrm{a})$ ). Regarding the singular values of the matrix $T_{n}\left(f^{(2)}\right)$, we observe again two sub-clusters of the same size (one at zero, the other at $\sqrt{2}$ ). The eigenvalues, as predicted by our results, are distributed 
Eigenvalue distribution of non-Hermitian multilevel block Toeplitz matrices 15

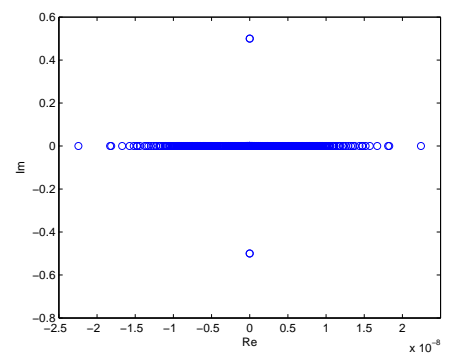

(a)

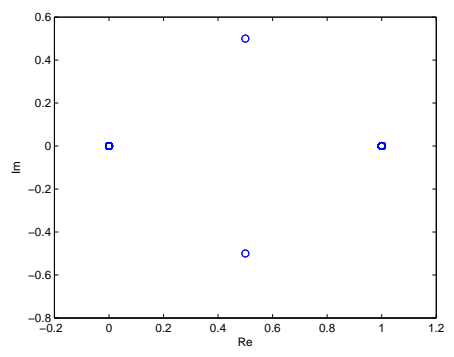

(b)

Figure 2. Eigenvalues in the complex plane for $n=400$ : (a) eigenvalues of $T_{n}\left(f^{(1)}\right)$, (b) eigenvalues of $T_{n}\left(f^{(2)}\right)$.
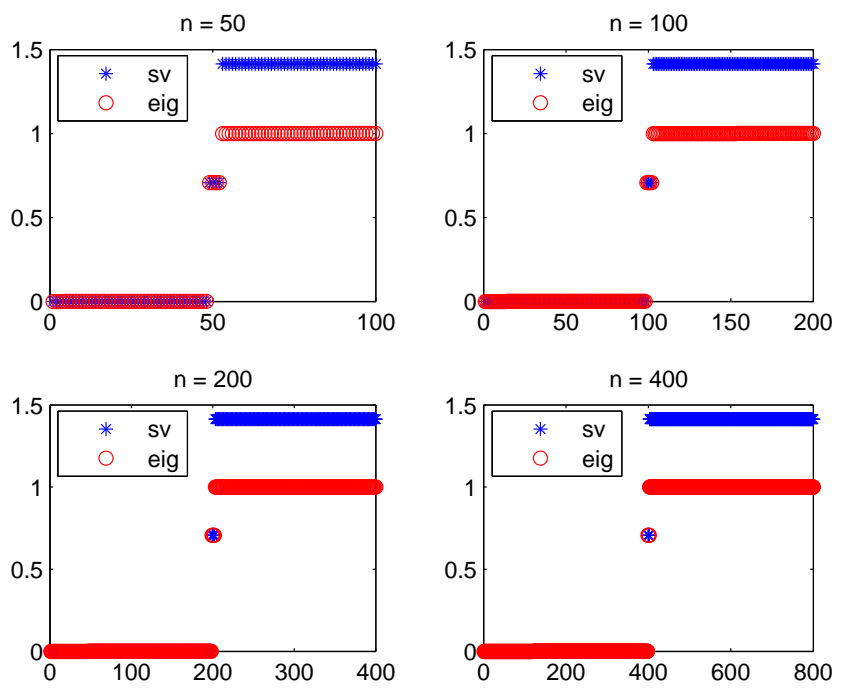

FIGURE 3. $T_{n}\left(f^{(2)}\right)$ : singular values and moduli of the eigenvalues in non decreasing order.

as the eigenvalues of the symbol, namely, half of them are around zero and half of them - around one (Figure 3). Observe, that also in this case, the range of the symbol is a strong cluster since the outliers are only four, again lying on the imaginary axis (Figure $2(\mathrm{~b})$ ).

Example 5.2 (Parametrized symbols). Consider

$$
B_{(c, r)}^{(3)}(t)=\left(\begin{array}{ll}
0 & 0 \\
1 & c+r e^{\mathbf{i} t}
\end{array}\right) \quad \text { and } \quad B^{(4)}(t)=\left(\begin{array}{ll}
0 & 0 \\
1 & 2-\cos (t)
\end{array}\right) .
$$


Let $T_{n}\left(f_{(c, r)}^{(3)}\right)$ and $T_{n}\left(f^{(4)}\right)$ be the corresponding block Toeplitz matrices with generating functions $f_{(c, r)}^{(3)}(t)=Q(t) B_{(c, r)}^{(3)}(t) Q(t)^{T}, t \in I_{1}$ and $f^{(4)}(t)=Q(t) B^{(4)}(t) Q(t)^{T}$. By varying the parameters $c$ and $r$ we simulate various spectral and singular value behaviour.
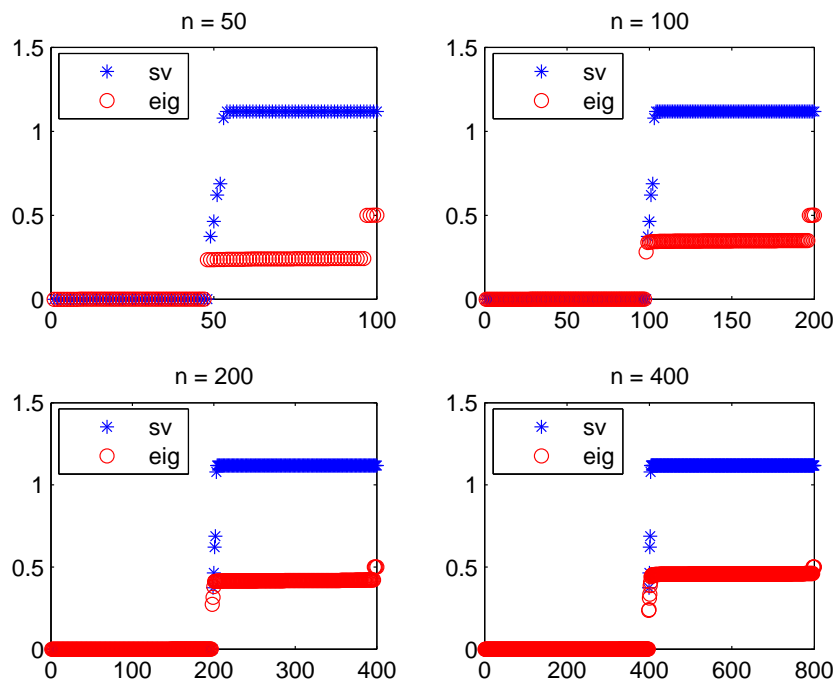

FIGURE $4 . \quad T_{n}\left(f_{\left(0, \frac{1}{2}\right)}^{(3)}\right)$ : singular values and moduli of the eigenvalues in non decreasing order.

The matrix $T_{n}\left(f_{(c, r)}^{(3)}\right)$ has two sub-clusters for the singular values, one at zero and one, as expected, at the range of $\sqrt{1+\left|c+r e^{\mathbf{i} x}\right|^{2}}$ (Figure 4 and Figure 5). Its eigenvalues, are one half equal to zero and one half - residing in a disc, centered at $c$ with radius $r$ (Figure 6). More precisely, most of the eigenvalues belonging to the second sub-cluster set (in the sense of Definition 2.2) stay very close to the frontier.

For the block Toeplitz matrix $T_{n}\left(f^{(4)}\right)$, both eigenvalues and singular values have two sub-clusters, one at zero and one in a positive interval (Figure 7 ). For the eigenvalues, as expected, the interval is $[1,3]$, which represents the range of the second eigenvalue symbol, namely, $2-\cos (t)$. For the singular values the interval is $[\sqrt{2}, \sqrt{10}]$, which represents the range of the second singular value symbol $\sqrt{1+(2-\cos (t))^{2}}$ (Figure 8).

Example 5.3 (Solution of linear systems). Consider now the solution of systems of linear equations with such matrices. Taking into account that the matrices are not Hermitian and our understanding of the spectral behavior, we use the GMRES 
Eigenvalue distribution of non-Hermitian multilevel block Toeplitz matrices 17
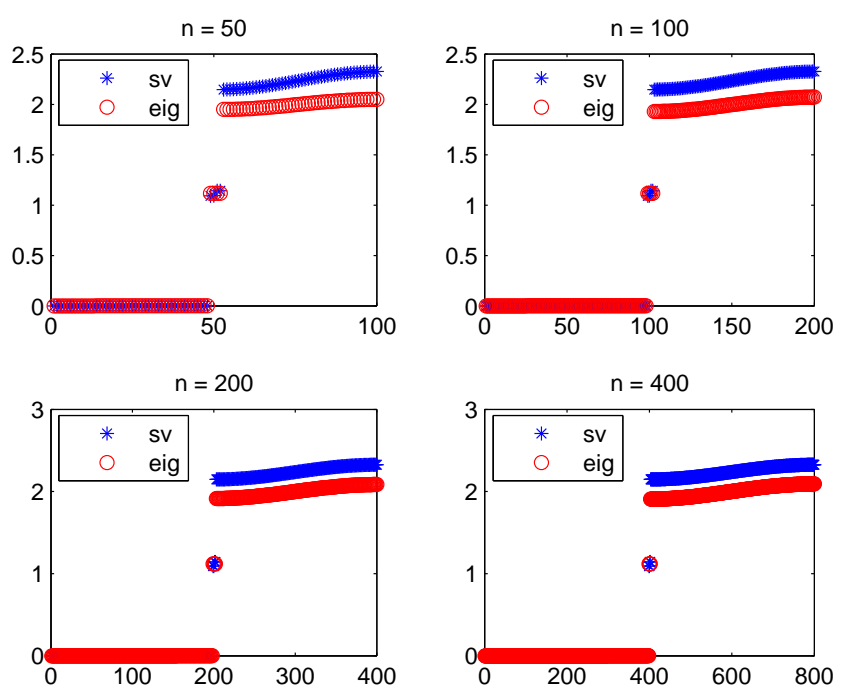

FIGURE $5 . \quad T_{n}\left(f_{\left(2, \frac{1}{10}\right)}^{(3)}\right)$ : singular values and moduli of the eigenvalues in non decreasing order.

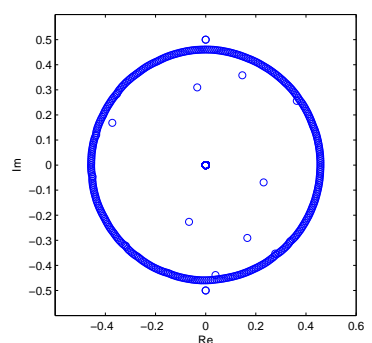

(a)

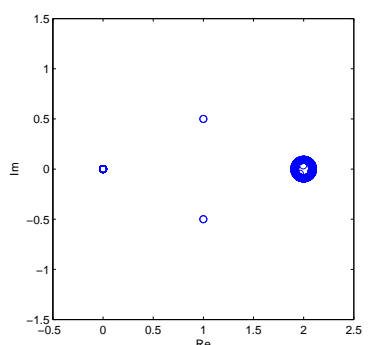

(b)

Figure 6. Eigenvalues in the complex plane for $n=400$ : (a) eigenvalues of $T_{n}\left(f_{\left(0, \frac{1}{2}\right)}^{(3)}\right)$, (b) eigenvalues of $T_{n}\left(f_{\left(2, \frac{1}{10}\right)}^{(3)}\right)$.

method (cf. [11]). We test matrices with two sub-cluster points $r_{1}$ and $r_{2}$ with $\left|r_{1}\right| \geq\left|r_{2}\right|$ and pose the question how the number of iterations depends on the size of the box containing $r_{1}$ and $r_{2}$. In addition, if these values are both real and positive, it is instructive to see how the number of iterations depends on $r_{1} / r_{2}$ (spectral conditioning). 

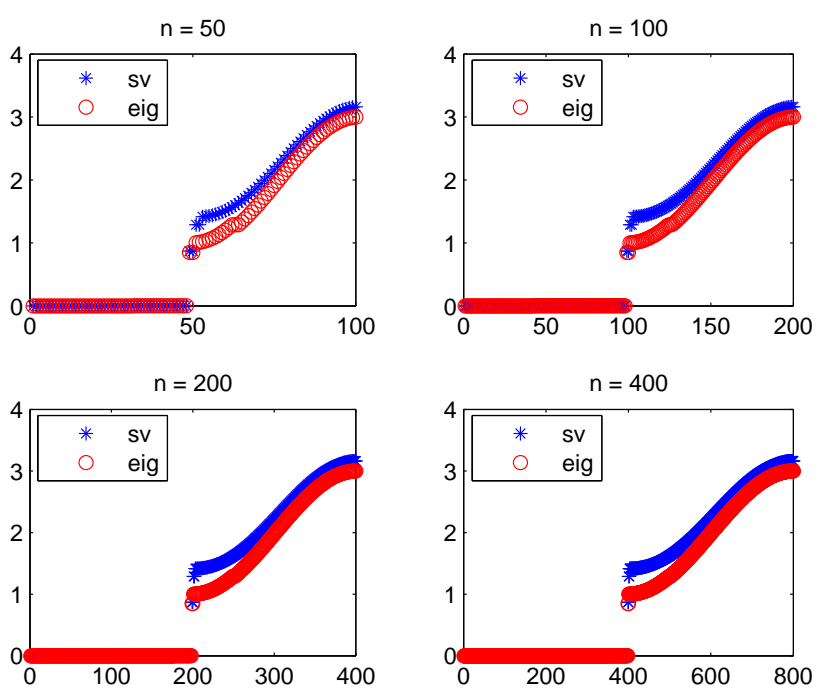

FiguRE 7. $T_{n}\left(f^{(4)}\right)$ : singular values and moduli of the eigenvalues in non decreasing order.

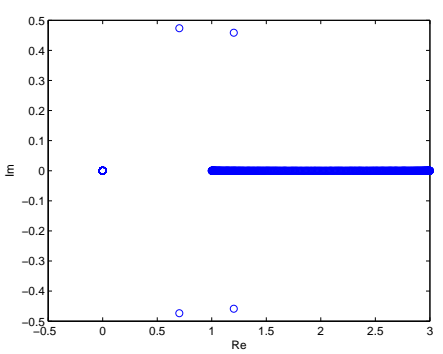

FIGURE 8. $T_{n}\left(f^{(4)}\right)$ : eigenvalues in the complex plane for $n=400$.

We begin by considering the symbol

$$
B_{\left(c_{1}, r_{1}, c_{2}, r_{2}\right)}^{(5)}(t)=\left(\begin{array}{ll}
c_{1}-r_{1} \cos (t) & 0 \\
1 & c_{2}+r_{2} e^{\mathrm{i} t}
\end{array}\right)
$$

and the corresponding matrix $T_{n}\left(B_{\left(c_{1}, r_{1}, c_{2}, r_{2}\right)}^{(5)}\right)$. The eigenvalues of $T_{n}\left(B_{\left(c_{1}, r_{1}, c_{2}, r_{2}\right)}^{(5)}\right)$ are divided in two sub-clusters, one in the interval $\left[c_{1}-r_{1}, c_{1}+r_{1}\right]$ and one in the disc centered at $c_{2}$ with radius $r_{2}$. We solve linear system with $T_{n}\left(B_{\left(c_{1}, r_{1}, c_{2}, r_{2}\right)}^{(5)}\right)$ and a random right-hand side. We apply full GMRES with a relative stopping tolerance $10^{-6}$ and use Matlab's built-in function gmres. 
Eigenvalue distribution of non-Hermitian multilevel block Toeplitz matrices 19

\begin{tabular}{c|ccccc}
$\backslash c_{2}$ & 5 & 15 & 25 & 35 & 45 \\
$n \backslash$ & & & & & \\
\hline 50 & 19 & 24 & 26 & 26 & 26 \\
100 & 19 & 24 & 25 & 26 & 26 \\
200 & 18 & 24 & 25 & 26 & 26 \\
400 & 18 & 24 & 25 & 26 & 26
\end{tabular}

TABLE 1. Number of GMRES iterations for $T_{n}\left(B_{\left(2,1, c_{2}, 1\right)}^{(5)}\right)$ varying $n$ and $c_{2}$.

\begin{tabular}{c|ccccc}
\begin{tabular}{c|c}
$\backslash r_{2}$ \\
$c_{2} \backslash$
\end{tabular} & 1 & 2 & 3 & 4 & 5 \\
\hline 10 & 30 & 35 & 40 & 45 & 53 \\
20 & 31 & 35 & 39 & 43 & 46 \\
30 & 31 & 35 & 38 & 41 & 43 \\
40 & 31 & 34 & 37 & 39 & 42 \\
50 & 32 & 35 & 37 & 39 & 41
\end{tabular}

TABLE 2. Number of GMRES iterations for $T_{200}\left(B_{\left(1+\mathbf{i}, 1, c_{2}, r_{2}\right)}^{(5)}\right)$ varying $c_{2}$ and $r_{2}$.

Table 1 shows the number of GMRES iterations required to reach the prescribed tolerance for varying $n$ and moving the center of the disc $c_{2}$. The other parameters are chosen as $c_{1}=2, r_{1}=1$, and $r_{2}=1$. We note, that the number of iterations is independent of the size of the system, $2 n$, however, it grows with increasing the spectral conditioning (the ratio $c_{2} / c_{1}$ ) until it reaches an asymptotic constant value (26 in this example). Table 2 reports the number of GMRES iterations for a fixed problem size, $n=200$, and moving the disc with the center $c_{2}$ and the radius $r_{2}$. The other sub-cluster is the complex segment $[\mathbf{i}, 2+\mathbf{i}$,$] . We note$ that the number of iterations grows when increasing the radius of the disc and decreases when moving the disc away from the origin, until it reaches a constant number, depending on both parameters $c_{2}$ and $r_{2}$. In other words, when the two sub-clusters are far away from each other, the convergence of GMRES is driven by the two sub-clusters, independently. When the two sub-clusters start to approach each other, they start to act as a unique clustered area and the convergence is accelerated.

Another perspective is given by Table 3, where the centers of the two subclusters are fixed but we increase their radii. As expected, the number of iterations increases more noticeably when increasing the radius of the sub-cluster closer to the origin. 


\begin{tabular}{c|ccccc}
\begin{tabular}{c|c}
$\backslash r_{2}$ \\
$r_{1} \backslash$
\end{tabular} & 1 & 2 & 3 & 4 & 5 \\
\hline 1 & 12 & 15 & 19 & 25 & 38 \\
2 & 13 & 15 & 19 & 26 & 39 \\
3 & 14 & 16 & 20 & 27 & 40 \\
4 & 15 & 17 & 21 & 28 & 41 \\
5 & 16 & 19 & 23 & 29 & 41
\end{tabular}

TABLE 3. Number of GMRES iterations for $T_{200}\left(B_{\left(10, r_{1}, 5+5 \mathbf{i}, r_{2}\right)}^{(5)}\right)$ varying $r_{1}$ and $r_{2}$.

\subsection{Examples with unbounded symbols}

Here we consider examples with unbounded symbols. The numerical results indicate that the main distribution result, that is Theorem 1.2, holds also under these weaker assumptions. However, the tools cannot be exactly the same: the Mergelyan Theorem requires the compactness and the compactness of the range does not hold if the symbol is unbounded. Probably some approximation arguments have to be introduced.

Let $\alpha(t)$ be the unbounded function

$$
\alpha(t)=\frac{1}{\sqrt{|t|}}, \quad t \in I_{1} .
$$

The function $\alpha(t)$ has an infinite Fourier series expansion, thus, for a fixed order $m$ it is approximated with its truncated series $\tilde{\alpha}(t)=\sum_{j=-m}^{m} \hat{\alpha}_{j} e^{\mathbf{i} j t}$. The Fourier coefficients $\hat{\alpha}_{j}$ are computed with high accuracy using the symbolic software package Mathematica.

Similarly to Subsection 5.1, for $s=2$, we consider the special classes of symbols $g$ of the form

$$
g(t)=Q(t) U(t) Q(t)^{T}, \quad t \in I_{1},
$$

where $Q(t)$ is defined in (31) but the choice of $U(t)$ varies. Since the behaviour of the singular values and eigenvalues of $T_{n}(g)$ does not change for large enough $n$, in the following examples we fix $n=100$.

Example 5.4. With

$$
U^{(1)}(t)=\left(\begin{array}{ll}
\mathbf{i} & 0 \\
\alpha(t) & 1
\end{array}\right),
$$

we find that the matrix $T_{n}\left(g^{(1)}\right)$ shows an unbounded maximal singular value, while the eigenvalues are divided in two sub-clusters - one at $\mathbf{i}$ and one at 1 (Figure 9). In other words, the unbounded character of the symbol does not play a role in the spectrum, which is determined only by the spectrum of the symbol. 
Eigenvalue distribution of non-Hermitian multilevel block Toeplitz matrices 21

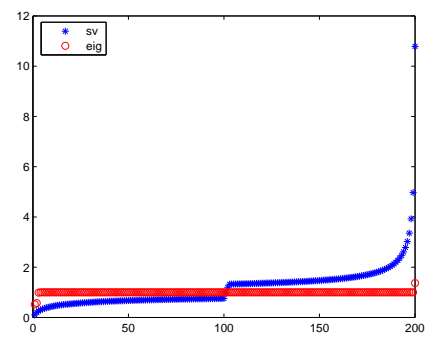

(a)

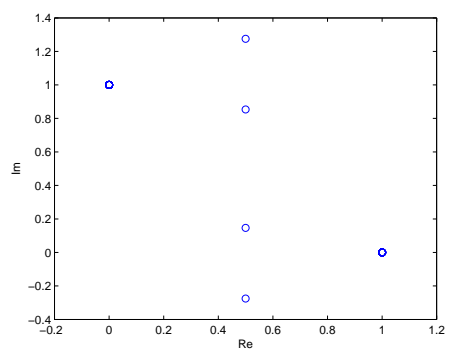

(b)

FiguRE 9. $T_{100}\left(g^{(1)}\right)$ : (a) singular values and moduli of the eigenvalues in non decreasing order. (b) eigenvalues in the complex plane.

Example 5.5. Consider

$$
U^{(2)}(t)=\left(\begin{array}{ll}
\alpha(t) & 0 \\
1 & \mathbf{i}
\end{array}\right) \quad \text { and } \quad U^{(3)}(t)=\left(\begin{array}{ll}
\alpha(t)(1+\mathbf{i} \cos (t)) & 0 \\
1 & 5 \mathbf{i}
\end{array}\right) .
$$

In these cases, the spectrum of the symbol is unbounded and in both cases, the spectrum and the singular values are distributed as the eigenvalues and the singular values of the symbol, correspondingly. The latter is clearly indicated in Figure 10 for the singular values of $T_{n}\left(g^{(2)}\right)$ and $T_{n}\left(g^{(3)}\right)$ and in Figure 11 for the eigenvalues of $T_{n}\left(g^{(2)}\right)$ and $T_{n}\left(g^{(3)}\right)$.

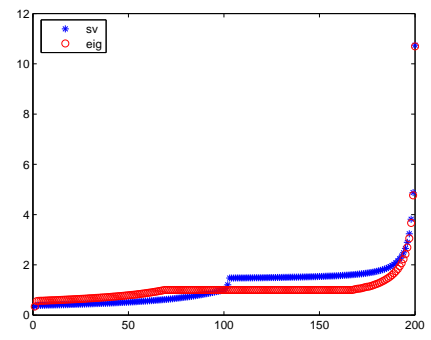

(a)

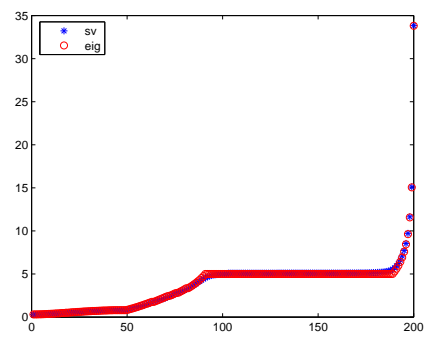

(b)

FIgURE 10. Singular values and moduli of the eigenvalues in non decreasing order of $T_{100}\left(g^{(2)}\right)$ in (a) and of $T_{100}\left(g^{(3)}\right)$ in (b).

We finally remark that the small number of large eigenvalues is expected by virtue of the distribution results, since the measure of the set, where the symbol $\alpha(t)$ is large, is indeed very small when compared with the measure $2 \pi$ of the domain $I_{1}$. 


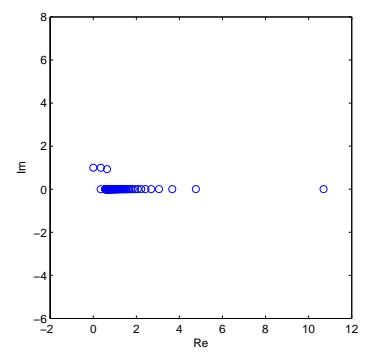

(a)

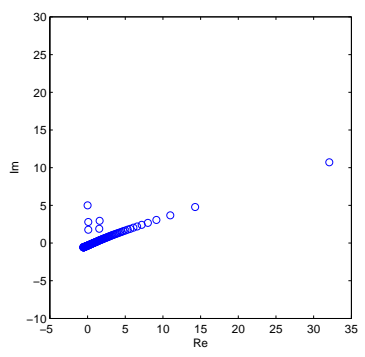

(b)

Figure 11. Eigenvalues in the complex plane: (a) eigenvalues of $T_{100}\left(g^{(2)}\right)$, (b) eigenvalues of $T_{100}\left(g^{(3)}\right)$.

\section{Concluding remarks and open problems}

As a conclusion, tools from approximation theory in the complex field (Mergelyan Theorem, see [10]), combined with those from asymptotic linear algebra $[21,22,14]$ are shown to be crucial when proving results on the eigenvalue distribution of nonHermitian matrix sequences. As stated in Remark 4.2, there are still open questions, some of which have been studied numerically in Section 5. An issue, with a significant practical importance, is related to constructing structured preconditioners. Thus, given a linear system with a block Toeplitz matrix with a symbol $f$, how to find a symbol $g$ such that (1) the spectrum of $g^{-1} f$ is well localized away from zero and as clustered as possible, and (2) a generic system with the matrix $T_{n}(g)$ is cheap to solve. The expectation is that the eigenvalues of $T_{n}^{-1}(g) T_{n}(f)$ are described asymptotically by the range of the eigenvalues of the new symbol $h=g^{-1} f$. Numerical tests in [5] seem to confirm the latter hypothesis in the distributional sense. We recall that in the case of Hermitian symbols such a result is rigorously proven both for the distribution and the localization (see [13] and references therein) and in the case of general non-Hermitian symbols - with respect only to the localization (see [9]). A future line of research should consider the extension of Theorem 1.2 for sequences $\left\{A_{n}\right\}$, where $A_{n}=T_{n}^{-1}(g) T_{n}(f)$ and where the role of the symbol in formula (2) is played by $h=g^{-1} f$. Some preliminary results can be found in [18]. A detailed study of the above open problems is a subject of future research.

\section{References}

[1] R. Bhatia, Matrix Analysis, Springer Verlag, New York, 1997.

[2] A. Böttcher, J. Gutiérrez-Gutiérrez, and P. Crespo, Mass concentration in quasicommutators of Toeplitz matrices, J. Comput. Appl. Math., 205 (2007), 129-148.

[3] A. Böttcher and B. Silbermann, Introduction to Large Truncated Toeplitz Matrices, Springer-Verlag, New York, 1999. 
Eigenvalue distribution of non-Hermitian multilevel block Toeplitz matrices 23

[4] F. Di Benedetto, M. Donatelli, and S. Serra-Capizzano, Symbol approach in a signalrestoration problem involving block Toeplitz matrices, in preparation.

[5] M. Donatelli, S. Serra-Capizzano, and E. Strouse, Spectral behavior of preconditioned non-Hermitian Toeplitz matrix sequences, in preparation.

[6] L. Golinskii and S. Serra-Capizzano, The asymptotic properties of the spectrum of non symmetrically perturbed Jacobi matrix sequences, J. Approx. Theory, 144-1 (2007), 84-102.

[7] U. Grenander and G. Szegö, Toeplitz Forms and Their Applications, Second Edition, Chelsea, New York, 1984.

[8] J. Gutiérrez-Gutiérrez, P. Crespo, and A. Böttcher, Functions of the banded Hermitian block Toeplitz matrices in signal processing, Linear Algebra Appl, 422-2/3 (2007), 788807.

[9] T. Huckle, S. Serra-Capizzano, and C. Tablino Possio, Preconditioning strategies for non Hermitian Toeplitz linear systems", Numerical Linear Algebra Appl., 12-2/3 (2005), 211-220.

[10] W. Rudin, Real and Complex Analysis, McGraw-Hill, New York, 1974.

[11] Y. Saad and M. H. Schultz, GMRES: a generalized minimal residual algorithm for solving nonsymmetric linear systems, SIAM J. Sci. Stat. Comput., 7 (1986), pp. 856-869.

[12] D. M. S. Santos and P. J. S. G. Ferreira, Reconstruction from Missing Function and Derivative Samples and Oversampled Filter Banks, Proceedings of the IEEE International Conference on Acoustics, Speech, and Signal Processing, ICASSP III (2004), 941-944.

[13] S. Serra-Capizzano, Spectral and computational analysis of block Toeplitz matrices with nonnegative definite generating functions, BIT, 39 (1999), 152-175.

[14] S. Serra-Capizzano, Spectral behavior of matrix sequences and discretized boundary value problems, Linear Algebra Appl., 337 (2001), 37-78.

[15] S. Serra-Capizzano, Generalized Locally Toeplitz sequences: spectral analysis and applications to discretized Partial Differential Equations, Linear Algebra Appl., 366-1 (2003), 371-402.

[16] S. Serra-Capizzano, The GLT class as a Generalized Fourier Analysis and applications, Linear Algebra Appl., 419-1 (2006), 180-233.

[17] S. Serra-Capizzano, D. Sesana, and E. Strouse, The eigenvalue distribution of products of Toeplitz matrices - clustering and attraction, Linear Algebra Appl., 432-10 (2010), 2658-2678.

[18] S. Serra-Capizzano and P. Sundqvist, Stability of the notion of approximating class of sequences and applications, J. Comput. Appl. Math., 219 (2008), pp. 518-536.

[19] P. Tilli, Singular values and eigenvalues of non-Hermitian block Toeplitz matrices, Linear Algebra Appl., 272 (1998), 59-89.

[20] P. Tilli, Locally Toeplitz matrices: spectral theory and applications, Linear Algebra Appl., 278 (1998), 91-120.

[21] P. Tilli, A note on the spectral distribution of Toeplitz matrices, Linear Multilin. Algebra, 45 (1998), pp. 147-159.

[22] P. Tilli, Some results on complex Toeplitz eigenvalues, J. Math. Anal. Appl., 239-2 (1999), 390-401. 
[23] E. Tyrtyshnikov and N. Zamarashkin, Spectra of multilevel Toeplitz matrices: advanced theory via simple matrix relationships, Linear Algebra Appl., 270 (1998), 15-27.

[24] J. Wilkinson, The Algebraic Eigenvalue Problem, Claredon Press, Oxford, 1965.

Marco Donatelli

Dipartimento di Fisica e Matematica,

Università dellInsubria,

Via Valleggio 11,

22100 Como, Italy

e-mail: marco.donatelli@uninsubria.it

Maya Neytcheva

Department of Information Technology

Uppsala University

Box 337

SE-751 05 Uppsala, Sweden

e-mail: Maya.Neytcheva@it.uu.se

Stefano Serra-Capizzano

Dipartimento di Fisica e Matematica,

Università dellInsubria,

Via Valleggio 11,

22100 Como, Italy

e-mail: stefano.serrac@uninsubria.it 